\title{
Spinal cord injuries in the 21st century
}

The following are excerpts from the Donald Munro Memorial Lecture delivered at the thirty-sixth Annual Conference of the American Paraplegia Society, September 4, 1990, Las Vegas, Nevada.

$$
\text { Phillip Harris, FRCSE, FRCPE, FRCS(glas), FRSE. }
$$

\section{The basic sciences and spinal cord injury}

The intense interest being shown in the experimental and clinical aspects of SCI has helped to stimulate an increasing activity in the basic sciences, and this involvement will surely escalate and further blossom in the future.

Research is progressing in special studies of the neuroanatomy of the spinal cord, for example, regarding circuitry and synaptic neuronal connections; and in time, a better understanding of the connections that different constituents make with one another will be achieved so that spinal cord circuitry may be related to physiology, chemistry and pharmacology. ${ }^{1}$

Ongoing research that will also continue and further develop include studies on neurotransmitters and the effects of various drugs that may mimic or block the effects of these chemicals, such as neuro-excitatory amino-acids. We will also have new chemotherapies for spasticity and for pain. Pharmacological studies in animals include methods of treating SCI to prevent 'the secondary injury'. Efforts to repair primary cord damage will be further avidly pursued. A recent important finding is that monosialic gangliosides appear to potentiate the action of endogenous trophic factors. ${ }^{2}$ Spectacular advances in the basic sciences are very likely; man is certainly 'a frontiers man,' full of curiosity and with many innovations.

Reprinted from the Journal of the American Paraplegia Society, vol 14, no. 2, April 1991, by kind permission of the American Paraplegia Society.

\section{Neuroimaging}

Remarkable advances are being made in the sphere of neuroimaging, and such studies are beginning to prove helpful to clinicians concerning the biomechanical and pathological changes that may occur as a result of spinal cord injury, in particular with the aid of computerised tomography (CT) and of magnetic resonance imaging (MRI). It is certain that further extensions of these techniques and newer advances will be forthcoming as the years go on, with less expensive but more sophisticated machines and shortened times for each patient's study. Obviously we must remember that the greatest vertebral column deformity, and therefore presumably also the greatest neural and vascular deformities, occur at the actual moment of impact when the accident occurs, and what is subsequently demonstrated by neuroimaging may often be different. MRI studies can help with the problem of spinal stability although this, up to now, remains a somewhat controversial topic.

Currently, reasonable information is available about deformation of the spinal cord, and of haemorrhages, necrosis, oedema and of cyst formation in the spinal cord, but these pathologies and others such as cord lacerations should in the future become much better defined with improved MRI techniques ....

MRI can also be of assistance regarding the prognosis of patients with acute spinal cord injuries. In a quite recent paper by Boudurant et al ${ }^{3}$ three appearances were noted: (a) a decreased signal intensity, consistent with an acute intraspinal haemorrhage (these patients did not show neurological improvement); (b) a bright signal 
intensity - cord oedema; and (c) a mixing of (a) and (b) - contusion. Patients who showed type (b) or (c) changes improved clinically. MR spectroscopy could assess vascular changes in the spinal cord and this technique will, hopefully, become available in the future.

Positron Emission Tomography (PET), at present, is a research tool for spinal pathology; but hopefully in the future it could become a practical clinical investigative technique, providing certain vital information, for example, on spinal cord blood flow and on the metabolism of the spinal cord.

\section{The spinal cord injury patient in the acute phase}

Hoped for advances in the optimal management of the traumatic spinal cord injury patient in the acute phase include the availability of more fully-trained, mobile paramedics and the transfer of patients promptly to a hospital situation with excellent facilities and properly trained staff ....

I am concerned about the amount of spinal surgery that is being carried out on some acute traumatic spinal cord injury patients. Most of the procedures advocated require considerable skill and experience, and several are not without risk to the patient .... In special situations there are definite indications for vertebral column surgery in patients with spinal cord injuries both in the acute phase and also in the later phases following injury. ${ }^{4}$ But the arguments and controversies continue and hopefully will be resolved in forthcoming years.

Will it be possible to control the neuropathological processes that develop following spinal cord injury? It has been shown in experimental animal studies that steroids may reduce cord odema and affect catecholamine production, improving spinal cord blood supply and with neurological benefit. In a recent important controlled study in May by Bracken et $a l^{5}$ on 487 patients, it was shown that those given a high dose of methylprednisolone (an intravenous bolus of $30 \mathrm{mg}$ per kilogram of body weight, followed by an infusion of $5.4 \mathrm{mg}$ per kilogram per hour for 23 hours) within 8 hours of the spinal cord injury showed more improvement in motor power and in sensory functions than the control patients whose treatment was given later, or who received either naloxone or placebo. It was felt that the high dose of methylprednisolone most probably inhibits lipid peroxidation and hydrolysis, thus retaining the cell membranes at the injury site. This is hopeful and should further stimulate those concerned to see that spinal cord injury patients are brought to a proper spinal unit expeditiously.

\section{Rehabilitation}

The Oxford dictionary definition of 'rehabilitation' is 'to restore to a previous condition' and there will be an increased demand for properly trained skilled rehabilitation personnel with a greater awareness of the value of therapies and benefits such as the utilization of technological advances such as microswitch control systems for higher level tetraplegics and computerised multichannel EMG biofeedback for spasticity, etc . ... It is known that more and more paralysed people resulting from spinal cord injuries are surviving to old age and that in many countries there is or will be an aging population. Soon, two-thirds of the world's population will be age 60 or over, certainly in the developing nations. Another problem is that there appears, as time goes on, to be less and less in the way of family support for disabled and elderly people, thus putting the onus on others. Financial and other resources will always be limited, and it would appear that even in a Utopian future, a balance will require to be struck between improving the functions of spinal cord injury patients and modifying their environment. Computerised database studies should help in these respects.

\section{Functional neuromuscular stimulation}

Functional neuromuscular stimulation (FNS) is one of the most exciting technical developments for spinal cord injury patients .... But there are many problems to be overcome, including system reliability, cost, 
energy loss, a fail-safe mode for patients, and a technique built in to prevent the patient from falling from the upright position ....

\section{Experimental animal studies and neural regeneration}

Progress is being made by many workers in the field of experimental animal studies and neural regeneration. A knowledge of developmental neurobiology of the central nervous system is necessary for future successful transplantation and regeneration.... Genes controlling growth and differentiation are now being identified, and Raisman and co-workers ${ }^{6}$ in the National Institute for Medical Research in London (ISRT 1987, 1989) are studying the effects of sending genes into a special damaged area and these genes then infiltrate appropriate cells. It is then necessary to reconstruct the complex connections required for the functioning of the central nervous system. According to Kakulas, ${ }^{7}$ 'The cure for spinal cord injury is a matter of de-depressing the genes responsible for the multiplication of neuroblasts, the sprouting of neurites and regrowth of axons and collaterals with the restoration of physiological connections and functionally useful reflexes by means of guided plasticity and synaptogenesis.' These and other research studies and new studies in the future obviously depend on the excellence of research workers and on the difficult problem of funding for basic reseach.

It is known that even in patients with a severe spinal cord injury, good functional recovery can occur if even a small number of axons survive.... In clinical situations in man, apart from traumatic spinal cord injury, there can, it is known, be good spinal neurological function recovery which may be temporary as in multiple sclerosis or permanent as in the Guillain-Barre syndrome, or in patients where spinal cord mechnical compression has been relieved .... Can we learn more from studies of such disorders regarding functional recovery after traumatic SCI? Even if the only remaining neurological function following the injury is just slight residual sensation, remarkable neurological recovery may en- sue, but can we come to recognise accurately predictive factors regarding functional outcome?

\section{Prevention}

In my dissertation today, I would emphasize that nothing is more important than the prevention of spinal cord injuries, both traumatic and non-traumatic, but much more is required by man to ensure that this aspect of spinal cord injury is seriously considered and given the publicity that it deserves....

Carefully planned, professional, high quality educational information programmes are required for the general public including school children and teenagers, educators, and legislators. This should include printed and audio-visual information. A nice balance between the emphasis of awareness of caution and of prevention is required so that the aspect of fear is not overstressed by such information.... The World Health Organization has recently constituted a committee concerned with prevention of neurotrauma . . .

\section{Training and education}

As I understand it, there is no ogranized training for those who wish to specialize in spinal cord injury work. Medical doctors become interested in this aspect of medicine in various ways, often associated with an early exposure and involvement in one of the main medical aspects of spinal cord injury patient care .... With a comprehensive centralised service in a main general teaching hospital including all relevant medical disciplines, laboratories, a library and research and teaching facilities, a core curriculum, and an organised course of instruction, suitable training would be obtained. At the end of training, some seal of recognition and application could be a diploma, or if possible, the training course would be linked to a university medical school so that a mastership could be attained .... Doctors and members of the professions complementary to medicine suitably trained in recognised centres will then become staff members and some will participate in spinal 
cord injury work in their own countries. Special consideration is necessary for the so-called developing countries regarding language, cultural links, finance, living accommodation, selection of trainees and the duration and type of training ....

\section{Conclusions}

Nearing the end of the 20th century which has indeed seen unparalleled advances in medicine and science, there still remain practical, serious problems concerning spinal cord injury and there are still many controversies. There is a speed and intensity in the way things are happening, but our main aims should be the prevention of spinal cord injury, the provision of excellent spinal services, plans for high quality independent living, and an encouragement to pursue research of excellence.

With database studies, modern computers, peer reviews, medical audits and demo- graphic studies, useful, reliable information is beginning to become available. As a result, there should be better use of available resources, greater efficiency by reducing costs and yet increased availability of services, better knowledge of clinical trials and of clinical work loads, and more in the way of accountability. Discarding inefficient, worthless or dangerous therapies and the introduction of new ones, problems of confidentiality and the capabilities of personnel will be carefully considered and acted upon. Medical audits could lead to the increased development of subspecialization and the appearance of more super-specialist with fewer generalists. ${ }^{8}$

Yes, we have an exciting, challenging future ahead of us; we cannot for a moment rest on our laurels.

(Copies of the full text of this address may be obtained by written request to the editor of the Journal of the American Paraplegia Society.)

\section{References}

1 Priestley JV (1987) Neuroanatomy of the spinal cord: current research and future prospects. Paraplegia 25: 198-204.

2 Hill RG (1987) Neuropharmacology of the injured spinal cord. Paraplegia 25: 209-11.

3 Boudurant FJ, et al (1990) Acute spinal cord injury: a study using physical examination and MRI. Spine 15: 161-68.

4 Gaines RW, Humphreys WG (1984) A plea for judgement in management of thoraco-lumbar fractures and fracture-dislocations: a reassessment of surgical indications. Clin Orthop 189: 36-42.

5 Bracken MB, Shepar MJ, Collins WF, et al (1990) A randomized controlled trial of methylprednisolone or naloxone in the treatment of acute spinal cord injury. New Eng J Med 322: 1405-1411.

6 Raisman G (1987) Genes and spine patients. International Spinal Research Trust Newsletter 12: 4-5. In: The Medical Research Intelligence Summary. London: ISRT, Vol 1, 1989.

7 Kakulas BA (in press) The applied neurobiology of human spinal cord injury: a review. Paraplegia.

8 Maurice-Williams RS (1990) Medical audit and neurosurgery. Brit J Neurosurgery 4: 83-86. 